E-Health ja, aber wie? Man hat uns versprochen, dass die Informatik unser Leben vereinfacht, aber dann haben wir erlebt, wie Behörden und Politik versuchen, alles unter ihre Kontrolle zu bringen - mit einer unglaublichen inflationären Bürokratie. Jetzt liegt es an uns, die weitere Entwicklung zu bestimmen!

Dr. med. Monique Gauthey,

Mitglied des FMH-Zentralvorstands, Verantwortliche für das Ressort eHealth

\title{
E-Health im Dienste der Patienten und Ärzte
}

Eigentlich wüssten wir doch, wie es sein sollte: Die für die Behandlung nötigen und wichtigen Patientendaten sind schnell und einfach verfügbar, keine ewige Sucherei mehr, keine Anrufe, um zu fragen, wo die Befunde sind, die Formulare sind vorausgefüllt - und doch scheint dies augenblicklich so weit weg.

Die Arbeitsgruppe eHealth der FMH hat sich die ehrgeizige Aufgabe gestellt, die zahlreichen Bemühungen auf dem Gebiet E-Health auf die Bedürfnisse von Patienten und Ärzten zu fokussieren. Die Arbeitsgruppe (AG) hat als ersten Schritt auf der Basis der strategischen Ziele der FMH für die Legislaturperiode 2008-2012 Vorschläge für eine E-Health-Strategie der FMH erarbeitet. Diese bewegen sich naturgemäss auf einem allgemeinen Niveau, sie sind mittelfristig orientiert. Als nächsten Schritt formuliert die AG in einem Positionspapier Standpunkte zu aktuellen E-Health-Themen wie etwa zu elektronischen Patientenakten, zu Versicherten- sowie zu Gesundheitskarten usw.

Im Mittelpunkt der E-Health-Strategie der FMH steht das Ziel, eine E-Health-Umgebung zu schaffen, die Patient und Arzt einen praktischen Nutzen bringt. Für eine aktive Mitgestaltung der Entwicklungen müssen wir nicht nur in den relevanten Gremien vertreten sein, wir müssen auch die Bedürfnisse der Ärzteschaft und der Patienten kennen, um sie nach aussen tragen zu können. Aus den Zielen und Bedürfnissen abgeleitet, können und müssen wir kontinuierlich die Positionen zu den verschiedenen aktuellen Themen erarbeiten.

Wichtig ist auch die Vernetzung der Mitglieder untereinander: Nicht jeder soll die zum Teil mühsamen Erfahrungen einer Einführung oder einer Umstellung selbst machen müssen! Häufig ist nicht das Werkzeug selbst entscheidend, sondern wie man dieses einsetzt. Auch die spätere Wieder- und Weiterverwendung der Daten kann sich je nachdem mehr oder weniger einfach gestalten.

Wir orientieren uns für die Erarbeitung der E-HealthStrategie der FMH an den folgenden Zielen:

\section{Patientensicherheit fördern}

E-Health muss immer die Patientensicherheit in den Vordergrund stellen und es müssen immer die Massnahmen getroffen werden, die geeignet sind, die Sicherheit der Patienten zu gewährleisten, z. B. bei der Datenspeicherung oder der Vermeidung von falschen Eingaben. Um- gekehrt können einzelne E-Health-Tools wie z. B. die elektronische Verschreibung gezielt für die Qualitätssicherung eingesetzt werden.

Behandlungsprozesse in den Mittelpunkt stellen Der Behandlungsprozess muss im Mittelpunkt stehen und unterstützt werden. E-Health-Tools sollten vor allem den Austausch von Informationen entlang der Behandlungskette unterstützen.

\section{Datenschutz, Privatsphäre und Autonomie sicherstellen}

Ein wirksamer Datenschutz und damit die Möglichkeit, die Privatsphäre zu wahren, bilden für uns die Voraussetzung, damit die Patienten und Ärzte E-Health akzeptieren. Ebenso müssen Patient und Arzt ihre Beziehung auch unter E-Health frei ausgestalten können, auch wenn diese sicher Veränderungen erfahren wird.

\section{Bildung anbieten}

Wir müssen E-Health und medizinische Informatik angemessen in die ärztliche Bildung integrieren. Dies bedeutet nicht, dass alle Ärztinnen und Ärzte Informatikspezialisten werden. Sie sollen jedoch durch die Vermittlung der notwendigen Kenntnisse und Fertigkeiten in der Lage sein, die verfügbaren Tools richtig einzusetzen. Patienten sollen über E-Health so weit Bescheid wissen, dass sie selbst entscheiden können, was mit ihren Daten geschieht. Ebenso setzt sich die E-Health-Strategie zum Ziel, eine qualitativ angemessene Vermittlung von medizinischem Wissen zu fördern.

\section{Finanzierung sicherstellen}

Last but not least ist die Finanzierung, insbesondere für die Infrastruktur in der Arztpraxis, entscheidend. Um den «Karren ins Rollen zu bringen» und eine kritische Masse auch in den Arztpraxen zu erreichen, braucht es über eine entsprechende Tarifierung hinaus unbedingt eine Anschubfinanzierung.

Werden diese Ziele erreicht, kommen wir in der Unterstützung der Patientenbehandlung sicher einen grossen Schritt weiter.

Judith Wagner, Leiterin eHealth FMH 